\title{
ACHIM STRUCHHOLZ
}

\section{Zusammenarbeit mit der Wirtschaft}

\section{Sehr geehrte Damen und Herren,}

ich darf mich herzlich für die Einladung bedanken, hier und heute als Fachfremder sprechen zu dürfen. Bitte erwarten Sie daher von mir keine großen Theorien zum Thema Sprachwissenschaft und Öffentlichkeit. Ich möchte lediglich über einige Erfahrungen berichten, die ich als Nicht-Experte in gemeinsamen Projekten mit Sprachwissenschaftlern gewonnen habe.

Vor rund eineinhalb Jahren haben wir als Team meines ehemaligen Arbeitgebers, der Kommuniktionsagentur Kohtes \& Klewes, gemeinsam mit Prof. Ludwig Jäger und seinem Diplomanden Kay Heiligenhaus von der RWTH Aachen, ein neues Beratungsprodukt mit dem Namen „Corporate Language" auf den Weg gebracht. Dahinter steht die Idee, dem unserer Meinung nach am meisten vernachlässigten Faktor der unternehmerischen Kommunikation zu mehr Gewicht zu verhelfen, eben der Sprache.

Corporate Language hat zum Ziel, über die abstrakte und theoretische Ebene hinaus konkrete Leitlinien und Maßnahmen zu entwickeln, wie Sprache zur Bildung und Weiterentwicklung von Unternehmenskultur und Unternehmensidentität eingesetzt werden kann. Wir haben dazu ein Instrumentarium entwickelt, das den zielgerichteten Einsatz von Sprache für unternehmerische Interessen nutzbar macht. Das heißt konkret: Sprache soll Unternehmen helfen, besser nach innen und außen zu kommunizieren, eine eigenständige Unternehmenskultur herauszubilden und damit in hartumkämpften Märkten erfolgreich zu sein.

Es gibt bereits einige Fälle, in denen Unternehmen, wenn wohl auch eher zufällig, versucht haben, Denkmuster durch neue Sprachmuster zu verändern und damit ihr Image und ihre Marktposition zu verbessern. Bei der Bahn sind aus Beförderungsfällen jetzt Fahrgäste geworden; den Telefonanschluß bei der Telekom gibt es nicht mehr untertänigst per Antrag, sondern durch Auftrag, und Energieversorger nennen ihre Kunden endlich Kunden und nicht mehr Benutzer.

Diese Beispiele zeigen, daß Unternehmen für die Bedeutung von Sprache sensibilisiert sind. Allerdings ist es bis zu einem strategischen, geplanten Einsatz von Sprache noch ein weiter Weg.

Wenn Sie sich vor Augen führen, daß deutsche Firmen pro Jahr schätzungsweise zwei Milliarden Mark allein für die Entwicklung von Corporate Design ausgeben, also für graphische Erkennungsmerkmale, und die Ausgaben für eine Art sprachliche Visitenkarte gegen null gehen, dann 
wird klar, daß der Nutzen von Sprache und Sprachwissenschaft noch dramatisch unterschätzt wird. Denn eigentlich ist es grotesk, daß in ein zentrales Feld der Kommunikation quasi nicht investiert wird.

Liegt das nun an den Unternehmen, die die Bedeutung von Sprache nicht erkennen? Oder an der Sprachwissenschaft, die die Berührung mit der Wirtschaft scheut und sich zudem im wissenschaftlichen Elfenbeinturm ganz bequem eingerichtet hat?

Ich denke, daß Sprachwissenschaft ihre Bedeutung für die Wirtschaft erst sehr zögernd erkennt, daß gerade bei Studenten noch große Berührungsängste existieren. Während für zahlreiche andere Fachbereiche der Umgang mit der Wirtschaft, zumindest aber mit marktwirtschaftlichen Mechanismen vom ersten Semester an selbstverständlich sind, sind Kooperationen zwischen Sprachwissenschaft und Ökonomie noch immer die Ausnahme.

Umgekehrt gilt übrigens dasselbe: Während Unternehmen wie selbstverständlich den Kontakt zu anderen Disziplinen suchen, gelten Linguisten gemeinhin noch als Exoten, über deren Arbeit man ohnehin nur diffuse Vorstellungen besitzt.

Unternehmen setzen noch kaum auf die Macht der Sprache. Hier liegt für die Linguisten also noch ein riesiges Betätigungsfeld, zumal an der Popularisierung der Disziplin angesichts zunehmend knapper öffentlicher Mittel ohnehin kein Weg vorbeiführt. Sprachwissenschaftler und Linguisten arbeiten in einem Themenfeld, daß Menschen zutiefst emotional berührt, wie die Diskussion um die Rechtschreibreform zeigt. Welcher Chemiker, Archäologe oder Betriebswirtschaftler kann das schon von sich behaupten?

Sprachwissenschaft kann also ein Wachstumsfeld der Zukunft werden. Gerade weil Unternehmen, Produkte und Märkte immer ähnlicher werden, steigt der Zwang zur Differenzierung. Und hier ist Sprache ein zentrales Element, Unternehmen von innen heraus zu verändern und damit letztlich am Markt erfolgreicher zu agieren.

Wer allerdings heute nach echten, wissenschaftlich fundierten Hilfen für den unternehmerischen Sprachalltag sucht, landet immer noch fast zwangsläufig bei rhetorischen Wunderheilern und Briefberatern aller Art. Bücher und Pseudo-Ratgeber zur schnellen und billigen Lösung von Sprach- und damit Kommunikationsproblemem gibt es zuhauf - ich würde mich freuen, wenn die Linguistik hier künftig echte Alternativen anbieten könnte.

Aus meinen Erfahrungen mit Corporate Language und langen, manchmal auch kontroversen Diskussionen möchte ich drei Thesen, nennen wir es ruhig drei Wünsche, an die Sprachwissenschaft formulieren:

1. Sprachwissenschaft muß ihre Ergebnisse besser vermarkten und den konkreten Nutzen ihrer Arbeit deutlich herausstellen. 
2. Es müssen mehr Sprachwissenschaftler in die Wirtschaft. Das heißt unter Umständen auch, das Lehr- und Ausbildungspläne praxisbezogener werden, als sie heute sind.

3. Universitäten und Institute müssen von sich aus mehr interdisziplinäre Projekte aktiv anbieten, um Unternehmen zur Zusammenarbeit zu gewinnen.

Aber auch die Wirtschaft muß selbstverständlich ihren Teil zu einer $\mathrm{Zu}$ sammenarbeit beitragen. Daher auch hier drei Thesen:

1. Die Wirtschaft muß sich bewußt werden, daß der ,weiche“ Gegenstand Sprache durchaus zu ,harten“ Ergebnissen, nämlich Markterfolg, führen kann.

2. Sprache braucht Zeit, um wirken zu können, funktioniert dann aber umso nachhaltiger. Die Wirtschaft braucht daher mehr Geduld für diese Prozesse und muß sich vom kurzfristigen Kosten-Nutzen-Denken lösen.

3. Unternehmer brauchen Mut, um auf klassische Kommunikationsinstrumente zugunsten von Sprachinstrumenten zu verzichten. Dies bedeutet auch eine Entscheidung für eine nachhaltige Kommunikationskultur und weg von kurzfristigen, aber eben auch kurzlebigen Kommunikationserfolgen. 